\title{
Partial Fanconi syndrome induced by ifosfamide
}

\author{
Muhammad Ajmal Panezai, MD (D), Charles Owen, MD, and Harold M. Szerlip, MD (D) \\ Division of Nephrology, Baylor University Medical Center, Dallas, Texas
}

\begin{abstract}
Ifosfamide-induced proximal tubular nephropathy can present as a spectrum of disease, from isolated hyperaminoaciduria to a partial or complete Fanconi syndrome. We report a case of ifosfamide-induced partial Fanconi syndrome in a man with metastatic progressive Ewing sarcoma and put forth a hypothesis on the mechanism.
\end{abstract}

KEYWORDS Acrolein; chloroacetaldehyde; Ewing sarcoma; Fanconi syndrome; hyperaminoaciduria; ifosfamide; Na-K-ATPase; nephrotoxicity; proximal tubulopathy

fosfamide is an alkylating agent that is a synthetic analog of cyclophosphamide. It is often used to treat a variety of cancers. Ifosfamide is associated with nephrotoxicity ranging from proximal tubular dysfunction to acute renal failure. Proximal tubular dysfunction, which has been reported in $1.4 \%$ to $5 \%$ of patients treated with ifosfamide, can present with a range of proximal tubule defects, from isolated aminoaciduria to symptomatic renal Fanconi syndrome affecting all proximal tubule transport including hydrogen ions, glucose, phosphorus, uric acid, and amino acids. ${ }^{1}$ Although it is believed that ifosfamide induces mitochondrial dysfunction and generalized energy depletion in the proximal tubules, it is unclear how such a defect would affect some transporters to a greater extent than other transporters. ${ }^{2-5}$

\section{CASE PRESENTATION}

A 52-year-old man with metastatic Ewing sarcoma was admitted to the hospital with a chief complaint of dyspnea. Computed tomography of the chest showed progressive metastatic nodules in the lungs. He began treatment with ifosfamide and etoposide. At the time of admission, his laboratory results showed a serum bicarbonate level of $31 \mathrm{mEq} / \mathrm{L}$; potassium, $3.6 \mathrm{mEq} / \mathrm{L}$; phosphorus, $2.5 \mathrm{mg} / \mathrm{dL}$; and glucose, $99 \mathrm{mg} / \mathrm{dL}$. Urine dip stick analysis did not reveal any glycosuria. The patient was treated with etoposide $125 \mathrm{mg} / \mathrm{m}^{2}$ body surface area and ifosfamide $2.5 \mathrm{~g} / \mathrm{m}^{2}$ body surface area for 4 days. He received a total of $20 \mathrm{~g}$ of ifosfamide. On day 3 of the regimen, he developed hypophosphatemia, hypokalemia, and glycosuria despite having a normal serum glucose.

Over the course of his hospitalization, the patient experienced a gradual worsening of serum electrolyte levels requiring large amounts of replacement potassium and phosphorus (Figure 1). His renal function and serum bicarbonate remained normal and his urine $\mathrm{pH}$ was $<6.0$ throughout the admission. A urine protein-creatinine ratio was $5.3 \mathrm{~g} / \mathrm{g}$ with a urine albumin-creatinine ratio of $329 \mathrm{mg} / \mathrm{g}$; 24-hour urine collection revealed excretion of $126 \mathrm{mmol}$ potassium, $2.2 \mathrm{~g}$ phosphorus $5338 \mathrm{mg}$ protein, and $343 \mathrm{mg}$ albumin. Urine amino acid quantitative test showed marked generalized aminoaciduria. Until his death on day 60 of the hospitalization, he required continued electrolyte supplementation.

\section{DISCUSSION}

Ifosfamide is an antineoplastic drug used in the treatment of soft tissue tumors in children and adults. Ifosfamide is mainly metabolized in the liver to its active metabolite 4hydroxy-ifosfamide, acrolein, and chloroacetaldehyde. ${ }^{4}$ However, ifosfamide can also be transported directly into proximal tubule cells via the organic cation transporter, where it can also be metabolized. ${ }^{2,6}$

In isolated mitochondria, chloroacetaldehyde inhibits mitochondrial complex 1, which would result in depletion of intracellular adenosine triphosphate (ATP). ${ }^{4-7}$ Because sodium-dependent transport across the apical membrane of the proximal tubule is indirectly linked to activity of the basolateral $\mathrm{Na}^{+}-\mathrm{K}^{+}$-ATPase, either depletion of ATP or direct inhibition of the ATPase could be responsible for impaired secretion of hydrogen ions and reabsorption of glucose, phosphate, and amino acids across the apical membrane of the proximal tubule.

Corresponding author: Harold M. Szerlip, MD, 3600 Gaston Ave., Suite 904 Barnett Tower, Dallas, TX 75246 (e-mail: Harold.Szerlip@BSWHealth.org) Color versions of one or more of the figures in this article can be found online at www.tandfonline.com/ubmc.

Received August 15, 2018; Revised October 5, 2018; Accepted October 11, 2018. 


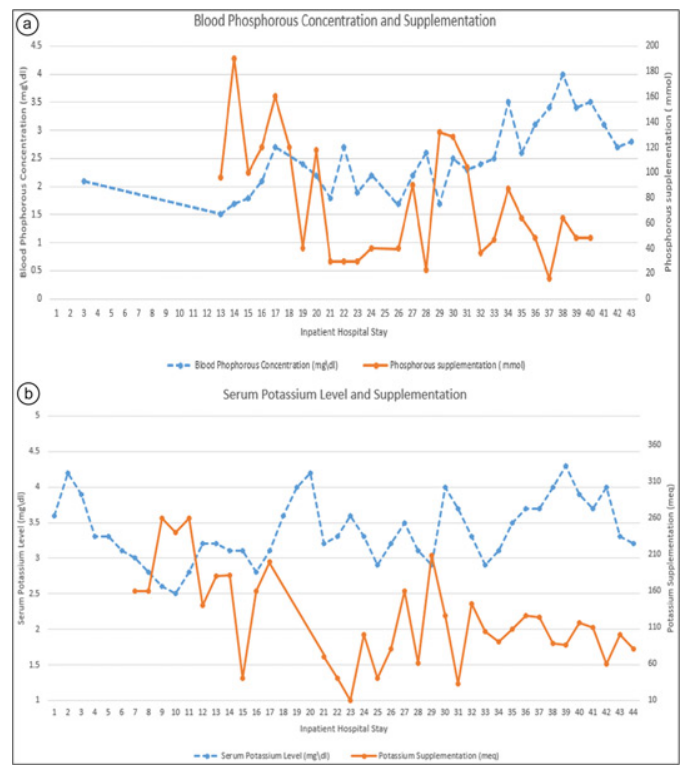

Figure 1. Laboratory values and supplementation throughout hospital course for (a) serum phosphorus and (b) serum potassium.

Ifosfamide-induced nephrotoxicity can present as a spectrum of proximal tubular dysfunction. Of patients who experience renal dysfunction, about $28 \%$ present with generalized hyperaminoaciduria, $17 \%$ present with severe impairment of both phosphate reabsorption and hyperaminoaciduria, $9 \%$ present with impairment of at least three substances, and only about 7\% present with full-blown renal Fanconi syndrome. ${ }^{8}$ Thus, ifosfamide tubulopathy can present with Fanconi syndrome in which there is a generalized defect in all sodiumdependent transport across the proximal tubule, including aminoaciduria, glycosuria with normal serum glucose, proximal tubular acidosis, and phosphate wasting or partial Fanconi syndrome in which there is an the absence of one or more cardinal features of Fanconi syndrome.

Our patient had partial Fanconi syndrome; his serum bicarbonate remained in the normal range and his urine never had evidence of bicarbonaturia. He required intravenous potassium and phosphorus supplementation throughout the course of his hospitalization. The reason why some proximal tubule transport processes are affected whereas others are not is not easily explained. Because all sodium-dependent transport across the apical membrane of the proximal tubule is indirectly driven by the basolateral $\mathrm{Na}^{+}-\mathrm{K}^{+}$-ATPase, it would be expected that any decrease in ATPase activity, whether caused by mitochondrial toxicity or direct toxicity of the ATPase, would decrease all proximal tubule transport processes.

The possible explanations for such a phenomenon include a differential effect of ifosfamide metabolites on the various transporters, the possibility that some of the transporters require less energy to function, or the possibility that ifosfamide toxicity affects some segments of the proximal tubule to a greater extent than other segments. It would be unlikely that ifosfamide would be more toxic to certain transporters. In addition, there is no evidence that the energy requirements of the various proximal transporters vary. The best explanation is that ifosfamide toxicity is not equal across the various segments of the proximal tubule. It has been shown that ifosfamide is transported into the proximal tubule cell by the organic cation transporter $2,{ }^{9}$ which is more abundant in the S3 segment of the proximal tubule. ${ }^{10}$ In addition, the S3 segment lies deeper within the kidney and is subject to more hypoxic stress. The sodium hydrogen exchanger is less abundant in the S3 segment, whereas amino acid and phosphorus cotransport are equally distributed throughout all segments of the proximal tubule. ${ }^{11}$ This could explain why aminoaciduria and phosphaturia are more common manifestations of ifosfamide nephrotoxicity than is generalized Fanconi syndrome.

This case demonstrates that Fanconi syndrome secondary to ifosfamide does not always present in its complete form but often presents with only partial transport defects. It is therefore important not to miss this diagnosis in a patient who has a normal bicarbonate.

\section{ORCID}

Muhammad Ajmal Panezai iD http://orcid.org/0000-0002-3697-1699 Harold M. Szerlip (iD http://orcid.org/0000-0002-3841-3172

1. Izzedine $\mathrm{H}$, Launay-Vacher V, Isnard-Bagnis C, Deray G. Druginduced Fanconi's syndrome. Am J Kidney Dis. 2003;41:292-309. doi:10.1053/ajkd.2003.50037.

2. Aleksa K, Halachmi N, Ito S, Koren G. A tubule cell model for ifosfamide nephrotoxicity. Can J Physiol Pharmacol. 2005;83:499-508. doi: 10.1139/y05-036.

3. Knouzy B, Dubourg L, Baverel G, Michoudet C. Targets of chloroacetaldehyde-induced nephrotoxicity. Toxicol. 2010;24:99-107. doi: 10.1016/j.tiv.2009.08.026.

4. Nissim I, Horyn O, Daikhin Y, et al. Ifosfamide-induced nephrotoxicity: mechanism and prevention. Cancer Res. 2006;66:7824-7831. doi:10.1158/0008-5472.CAN-06-1043.

5. Springate J, Chan K, Lu H, Davies S, Taub M. Toxicity of ifosfamide and its metabolite chloroacetaldehyde in cultured renal tubule cells. In Vitro Cell Dev Biol Anim. 1999;35:314-317. doi:10.1007/s11626999-0080-y.

6. Woodland C, Ito S, Granvil CP, Wainer IW, Klein J, Koren G. Evidence of renal metabolism of ifosfamide to nephrotoxic metabolites. Life Sci. 2000;68:109-117.

7. Lind MJ, McGown AT, Hadfield JA, Thatcher N, Crowther D, Fox BW. The effect of ifosfamide and its metabolites on intracellular glutathione levels in vitro and in vivo. Biochem Pharmacol. 1989;38:1835-1840.

8. Rossi R, Pleyer J, Schafers P, et al. Development of ifosfamideinduced nephrotoxicity: prospective follow-up in 75 patients. Med Pediatr Oncol. 1999;32:177-182.

9. Ciarimboli G, Holle SK, Vollenbröker B, et al. New clues for nephrotoxicity induced by ifosfamide: preferential renal uptake via the human organic cation transporter 2. Mol Pharmaceutics. 2011;8: 270-279. doi:10.1021/mp100329u.

10. Karbach U, Kricke J, Meyer-Wentrup F, et al. Localization of organic cation transporters OCT1 and OCT2 in rat kidney. Am J Physiol Renal Physiol. 2000;279:F679-F687. doi:10.1152/ajprenal.2000.279.4.F679.

11. Zhuo JL, Li XC. Proximal nephron. Compr Physiol. 2013;3: 1079-1123. 